ISSN Print: 2076-1449,ISSN Online: 2076-9539

International Journal of

Wireless and Microwave Technologies

\title{
IJWMT Kol.2
}

http://www.mecs-press.org/

Vol.2 No.6 December 2012 


\section{International Journal of Wireless and Microwave Technologies (IJWMT)}

Volume 2, Number 6, December 2012

\section{Contents}

Design and Simulation Implementation of an Improved PPM Approach

Guo Fan, Feng Bo, Yu Min

A New Solution of Multicast Packets Management for Managed Ethernet Switch

Sheng $L U$

Software Defect Detection-oriented Static Analysis Techniques

Hиа ZHANG

Study on System Architecture Design of University Students Quality Evaluation

He Yongqiang, Wang Jiaxin

TCP/IP Jumbo Frames Network Performance Evaluation on A Test-bed Infrastructure

Shaneel Narayan, Paula Raymond Lutui

Evaluation of Performance for Wireless Sensor Networks Based on Gray Theory

JING Jun li, YANG Jie

Wireless Multi-hop Network Scenario Emulation with MinGenMax Error Based on Interval

Equivalent Character of Wireless Communication

Huizhou Zhao

A Wireless Solution to Collecting and Displaying Oil Temperature Data Based on Zigbee Network

Cui Jingcong, Chen Lidong,Li Xunming,Huang Chunhai

A Source-Based Multicast Scheme in IEEE 802.16 Mesh Mode

Mortaza Maleki,Saleh Yousefi,Ali Azarpeyvand

An Epistemic Model Checking Approach to Web Service Compositions 